\title{
A novel CRISPR activation mouse enables modelling of aggressive lymphoma and interrogation of venetoclax resistance.
}

Marco Herold ( $\nabla$ herold@wehi.edu.au )

Walter and Eliza Hall Institute of Medical Research

\section{Yexuan Deng}

Walter and Eliza Hall Institute of Medical Research

\section{Sarah Diepstraten}

Walter and Eliza Hall Institute of Medical Research

\section{Margaret Potts}

Walter and Eliza Hall Institute of Medical Research https://orcid.org/0000-0001-9796-9683

Amy Heidersbach

Genentech Inc

\section{Andrew Kueh}

Walter and Eliza Hall Institute of Medical Research

\section{Martin Pal}

Garvan Institute of Medical Research

\section{Göknur Giner}

The Walter and Eliza Hall Institute of Medical Research

\section{Stephen Wilcox}

Walter and Eliza Hall Institute of Medical Research

\section{Lin Tai}

Walter and Eliza Hall Institute of Medical Research

\section{Andreas Strasser}

The Walter and Eliza Hall Institute of Medical Research https://orcid.org/0000-0002-5020-4891

\section{Benjamin Haley}

\author{
Genentech
}

\section{Quan Zhao}

Nanjing University https://orcid.org/0000-0002-8533-1938

\section{Gemma Kelly}

Walter and Eliza Hall Institute of Medical Research

\section{Article}


Keywords: CRISPRa, double hit lymphoma, BCL-2

Posted Date: September 10th, 2021

DOI: https://doi.org/10.21203/rs.3.rs-856034/v1

License: (c) (1) This work is licensed under a Creative Commons Attribution 4.0 International License. Read Full License

Version of Record: A version of this preprint was published at Nature Communications on August 12th, 2022. See the published version at https://doi.org/10.1038/s41467-022-32485-9. 


\section{Abstract}

CRISPR technologies have advanced cancer modelling in mice, but CRISPR activation (CRISPRa) methods have not been thoroughly exploited in this context. Here we establish a CRISPRa mouse $\left(\mathrm{dCas} 9 \mathrm{a}-\mathrm{SAM}^{\mathrm{KI} /+}\right.$ ) for inducing gene expression in vivo and in vitro. Using dCas9a-SAM ${ }^{\mathrm{KI} / \mathrm{KI}}$ primary lymphocytes, we induced B cell restricted genes in the T cell lineage and vice versa, demonstrating the power of this system. Next, to model double hit lymphoma (DHL), we transactivated pro-survival BCL-2 in $\mathrm{E \mu}-\mathrm{Myc}^{\mathrm{T} /+} ; \mathrm{dCas} 9 \mathrm{a}-\mathrm{SAM} \mathrm{K}^{\mathrm{KI} /+}$ haematopoietic stem and progenitor cells. Lethally-irradiated mice transplanted with these cells rapidly developed lymphomas expressing high BCL-2. Unlike standard E $\mu-$ Myc lymphomas, BCL-2-expressing lymphomas were highly sensitive to the BCL-2 inhibitor venetoclax. Finally, we performed genome-wide activation screens in these lymphoma cells and found a dominant role for the BCL-2 family protein A1 in venetoclax resistance. This demonstrates the power of our CRISPRa model for mimicking disease and provides insights into potential resistance mechanisms towards targeted therapies.

\section{Introduction}

The CRISPR/Cas9 system is an elegant tool for genomic engineering. While initial usage was restricted to mutation induced loss-of-function applications, recent advances have facilitated more sophisticated forms of genetic manipulation. One example is the CRISPR activation (CRISPRa) system which utilises a dead Cas9 (dCas9) variant lacking enzymatic activity, thereby converting Cas9 to an inert DNA-binding protein scaffold. ${ }^{1}$ Fusion of a transcriptional VP $64^{2}$ activation domain to dCas 9 produces a transcriptional activator, which can be targeted to any promoter of any gene through a sequence specific single guide RNA (sgRNA) in the presence of a protospacer adjacent motive (PAM, NGG) located downstream of the sgRNA binding sequence. ${ }^{3,4,5}$ Early reports showed promising results for gene activation by dCas9-VP64 at some genetic loci, but low to no gene induction at others. ${ }^{3,4,5,6}$ This inconsistency across different genomic loci, sparked the development of more potent dCas 9 transactivators. $7,8,9,10$ One such effective CRISPRa systems is the Synergistic Activation Mediator (SAM). It relies on the dCas9-VP64 fusion protein and two additional transcriptional activator domains, p65 and HSF1, which can be recruited into the complex by the MS2 RNA binding protein through loops in the sgRNA scaffold, leading to strong induction of expression from targeted genes. ${ }^{9}$

An exciting application of CRISPRa is the potential to model complex human diseases. ${ }^{11}$ Cancer is a highly heterogenous disease with many different pathways deregulated within the same cancer type. ${ }^{12}$ The ability to create pre-clinical disease models that faithfully reflect the hallmarks of human disease is critical for the identification of specific cancer drivers and/or therapy resistance factors. ${ }^{13}$ However, mouse models that accurately mimic aggressive lymphomas, such as double hit lymphoma (DHL), an aggressive subset ( $10 \%$ ) of diffuse large B cell lymphomas that express high levels of both MYC and BCL-2 due to chromosomal translocations, are lacking. ${ }^{14,15}$ Previous attempts to model this disease

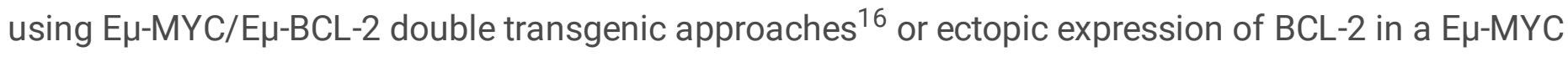


transgenic background ${ }^{17}$ failed to recapitulate $\mathrm{DHL}$, instead giving rise to progenitor tumours; probably due to unnaturally high expression of both gene products. Hence, a CRISPRa approach represents a potential solution as gene expression is induced from endogenous loci at more moderate levels.

Here we demonstrate the utility of a new powerful CRISPRa mouse model and applicability in primary cells of the haematopoietic system. Initially, we show gene induction in primary B and T cells derived from the dCas9a-SAM ${ }^{\mathrm{KI} / \mathrm{KI}}$ mice transduced with sgRNAs targeting genes such as CD19 and CD4. While these genes can be further induced in their respective lineages, impressively we are also able to express $B$ cell specific genes in the T cell lineage and vice versa. To test the suitability of our CRISPRa mice for generating disease models, we induced MDM2 expression (the natural antagonist of the tumour suppressor p53) in haematopoietic stem and progenitor cells (HSPCs) from CRISPRa enabled E $\mu-M y C$ transgenic mice. Following transplantation of these cells into lethally irradiated mice, accelerated tumour onset was observed. Having validated the strong capability of this CRISPRa system, we set out to develop a model of DHL. This was achieved through activation of endogenous BCL-2 expression in E $\mu$ Myc/dCas9a-SAM ${ }^{\mathrm{KI} /+}$ HSPCs and transplantation into lethally irradiated mice. Rapid and aggressive lymphomas develop that are characterised by CD19/B220 double positive tumour cells expressing high cMYC and BCL-2, which are markers of human $\mathrm{DHL}^{18}$. These lymphomas are highly sensitive to treatment with venetoclax, the BH3-mimetic drug that specifically binds and inhibits BCL2. Venetoclax is FDA approved for Chronic Lymphocytic Leukemia and Acute Myeloid Leukemia (AML) and is in clinical trials for several other cancers. ${ }^{19}$ Identifying resistance factors to venetoclax therapy is therefore an area of great clinical relevance. Whilst CRISPR gene knock-out screens have identified TP53 loss and other factors that are mediators of venetoclax resistance, ${ }^{20,21}$ no studies have utlised CRISPRa to investigate genes that can confer venetoclax resistance when upregulated from their endogenous promoters. Cell lines derived from the CRISPRa mouse model of DHL that are highly sensitive to venetoclax provide an ideal platform to perform genome-wide CRISPRa screens for identifying resistance factors. These screens revealed a dominant role for the understudied pro-survival protein A1 (called BFL-1 in humans) in venetoclax resistance.

\section{Results}

\section{Establishment of a robust CRISPR activation platform}

CRISPRa has the potential to be used for the development of accurate disease models because the induction of oncogenic driver genes from their endogenous promoters can be more modest and physiologically relevant compared to that achieved with cDNA constructs. We therefore sought to establish a robust and widely applicable CRISPRa system for the generation of faithful pre-clinical cancer models and the identification of targets that could translate into improved therapies for cancer patients. We adapted the SAM system originally described in a two-vector configuration to be expressed from a single construct to achieve similar expression of all the components required for CRISPR mediated gene induction. ${ }^{9}$ To this end, we linked the dCas9-VP64 via a T2A sequence to the MS2-p65-HSF1 expression 
cassette (Fig. 1a). In addition, we incorporated a GFP sequence as a marker via a second T2A sequence downstream of the dCas9-VP64-T2A-MS2-p65-HSF1 coding sequence. We incorporated the cassette into a lentiviral vector for ease of manipulating cells ${ }^{22}$. We initially sought to validate the efficiency of the CRISPRa cassette in vitro. We therefore introduced lentiviral vectors encoding the CRISPRa cassette and one of three unique sgRNAs targeting the BCL-2 promoter into cell lines derived from the $E \mu-M y C$ transgenic mouse model of lymphoma ${ }^{23}$ (Supplementary Fig. 1). Western blot analysis of two independent lymphoma cell lines confirmed that all sgRNAs caused a substantial increase in BCL-2 expression, detectable even before puromycin selection of sgRNA-transduced cells (Fig. 1b).

$E \mu-M y c$ lymphomas are highly reliant on the pro-survival protein MCL-1 for their sustained survival ${ }^{24,25,26}$ and hence the elevated BCL-2 expression increased resistance to the MCL-1 selective inhibitor S63845 (Fig. 1c). Interestingly, the enforced expression of BCL-2 in these lymphoma cells did not sensitise them to the BCL-2 inhibitor venetoclax (Fig. 1d), likely because these tumour cells did not develop under conditions of high BCL-2 expression and therefore do not depend on BCL-2 expression for continued survival. These experiments confirmed that our CRISPRa cassette could induce strong upregulation of genes in cell lines in vitro but also highlighted the limitations of engineering established cell lines for studies relevant to more sophisticated cancer models. We therefore sought to progress this system to an in vivo setting, by generating transgenic mice with a similar configuration for CRISPRa mediated transcriptional upregulation.

\section{Generation of CRISPRa transgenic mice}

For flexible expression of the CRISPRa system in vivo, we targeted the dCas9-VP64-T2A-MS2-p65-HSF1 cassette into the ubiquitously expressed Rosa26 locus. We used the CTV vector, ${ }^{27}$ which has a loxP flanked stop cassette between the CAGS promoter and the CDNA, allowing for temporal or cell type specific induction of gene expression upon CRE mediated deletion (Fig. 2a). Crossing the CRISPRa transgenic mice to a CRE deleter strain removed the stop cassette, allowing expression of the CRISPRa components in all tissues. We refer to the resulting strain as dCas9a-SAM. Transgene insertion and expression was confirmed by PCR on DNA isolated from mouse tails and flow cytometric analysis for GFP on cells isolated from the thymus, spleen, bone marrow and lymph nodes (Fig. 2b, c). Moreover, intracellular staining and flow cytometric analysis of thymus and bone marrow cells with Cas9-specific antibodies confirmed that the CRISPRa components were expressed in vivo (Fig. 2d). Since the dCas 9 protein is fused to the VP64 activation domain, we next sought to determine whether the constitutive expression of a transcriptional activator protein had any impact on the cellular composition of the mice. We analysed the haematopoietic compartment because it is particularly sensitive to cytotoxic stress and even subtle changes in gene expression. We found that homozygous $d C a s 9 a-S A M$ mice showed no differences in the frequencies and numbers of the different immune cell subsets in the thymus, bone marrow, spleen, and lymph node compared to wildtype mice (Supplementary Fig. 2). Importantly, aged (older than 12 months) dCas9a-SAM ${ }^{K I / K I}$ mice showed no signs of disease, further substantiating that 
constitutive expression of the CRISPRa system in all tissues of the animals does not cause marked toxicity or substantive changes to the proportions of all tested cell types.

Next, we assessed the efficiency of the CRISPRa components for gene activation in primary cells derived from the $d$ Cas9a-SAM mice. To this end, we stimulated splenocytes isolated from dCas9a-SAM ${ }^{K I / K I}$ mice with LPS or Concanavalin A plus Interleukin-2 for the generation of activated B or T cell blasts, respectively. We transduced these cells with the $B c /-2$ sgRNA1 construct (that co-expresses puromycin resistance) and selected transduced cells with puromycin for 2 days. Analysis of BCL-2 expression by intracellular flow cytometry and Western blotting revealed upregulation of BCL-2 protein in both $B$ and T cells (Fig. 3a). To assess the potential of the CRISPRa system for targeting various genes, we introduced sgRNAs for CD19 or IRF4 into B cells. As expected, CD19-specific sgRNAs elevated surface expression of CD19 above the basal level, while introducing sgRNAs for IRF4, known to induce differentiation of B cells into plasma cells, ${ }^{28}$ enhanced the frequency of cells positive for the plasma cell marker CD138 (Syndecan) (Fig. 3b, c). Similarly, transducing activated T cells with sgRNAs targeting the CD4 promoter enhanced expression of CD 4 on the cell surface (Fig. 3d). Since these genes are already transcriptionally active in B cells or T cells, respectively, we next challenged our CRISPRa system by attempting to induce expression of genes that are normally transcriptionally silent in these cells. We first introduced sgRNAs targeting the B cell marker CD19 into T cells. Remarkably, this elicited CD19 expression in almost $50 \%$ of T cells (Fig. 3e). Similarly, introduction of sgRNAs targeting the T cell specific CD4 gene promoter into B cells resulted in B cells with CD4 expression (Fig. 3f). These data demonstrate that we have developed a powerful CRISPRa mouse model that displays no detectable toxicity as well as the potency required to induce targeted gene expression in primary cells, even of genes that are normally silenced within a specific fully differentiated cell type.

\section{Exploiting CRISPRa in vivo for the development of aggressive lymphomas}

Having validated the efficiency of the CRISPRa mouse for gene induction in primary cells, we set out to test its applicability for developing disease models. Initially, we sought to confirm whether induction of a gene product by CRISPRa could indeed affect disease aetiology, for example, by modulating the latency of tumour development. We know from previous reports that deleting the p53 tumour suppressor accelerates $E \mu$-Myc-driven tumourigenesis. ${ }^{29}$ To replicate the effect of loss of p53 activity using CRISPRa, we transduced $E \mu-M y c / d C a s 9 a-S A M^{K I /+} H S P C s$ with sgRNAs to induce expression of MDM2 (an E3 ligase that degrades $\mathrm{p} 53$ protein) ${ }^{30}$ (sgMdm2), or non-targeting sgRNAs as controls. The transduced HSPCs were transplanted into lethally irradiated C57BL/6-Ly5.1 recipient mice which were observed for tumour development (Fig. 4a and Supplementary Fig. 3a). As occurs for p53 knockout in the E $\mu$-Myc background, we observed accelerated tumour onset in the mice reconstituted with $E \mu-M y c / d C a s 9 a-S A M M^{K I /+} / s g M d m 2$ transplanted HSPCs compared to controls. To determine the levels of p53 in the lymphomas expressing 
sgMdm2, we derived cell lines and induced expression of p53 with the MDM2 inhibitor Nutlin3a. ${ }^{31}$ Western blot analysis clearly demonstrated a reduction in p53 protein levels in EM-Myc/dCas9a$S A M^{K I /+} / s g M d m 2$ lymphomas compared to controls consistent with elevated MDM2 levels (Supplementary Fig. 3b). Having shown that our CRISPRa model is indeed powerful enough to affect lymphomagenesis by induced expression of MDM2 leading to reduced p53, we next sought to develop a long sought after model of aggressive $\mathrm{DHL}$, for which all previous attempts to mimic this devastating disease have failed. Such a lymphoma model would facilitate the identification and validation of novel therapeutic strategies for patients with DHL. To this end we used the same approach as described above, this time using one of the validated $\mathrm{sgBCl}-2$ constructs described in Fig. 1. Irradiated mice were injected

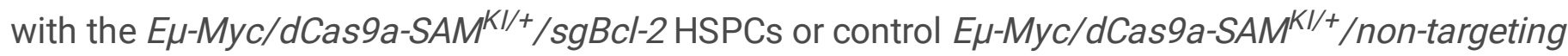
sgRNA HSPCs, and 6 weeks post transplantation, BCL-2 expression was analysed in haematopoietic cells of the mice by flow cytometry. The analysis revealed increased BCL-2 expression in peripheral blood cells of mice transplanted with $E \mu-M y c / d C a s 9 a-S A M^{K I /+} / s g B c l-2$ HSPCs (Fig. 4b). Accordingly, these mice went on to develop aggressive lymphomas with a median latency of 68 days, compared with a median latency of 132 days for mice transplanted with the same HSPCs that had been transduced with a nontargeting sgRNA (Fig. 4c). Characterisation of $E \mu-M y c / d C a s 9 a-S A M K l /+/ s g B c l-2$ lymphomas revealed a B cell phenotype (CD19/B220 double positive; Fig. 4d) and high BCL-2 protein expression (Fig. 4e), which are both also observed in human $\mathrm{DHL}^{18}$. These mature $\mathrm{B}$ cell lymphomas could readily be derived into cell lines in vitro (Fig. 5). This model of aggressive lymphoma therefore contrasts with a previous one that utilised $E \mu-M y c / E \mu-B c l-2$ double transgenic mice which developed lymphomas exhibiting an immature haematopoietic progenitor phenotype ${ }^{16}$ that could not be grown in vitro ${ }^{32}$ (Fig. 4, 5).

For further characterisation and experimentation, cell lines were derived from the $E \mu-M y c / d C a s 9 a-$ $S A M^{K I /+} / \mathrm{sg} B C l-2$ lymphomas and non-targeting control lymphomas. All sgBCl-2 tumour-derived lines displayed high expression of BCL-2 and the pro-apoptotic BH3-only protein BIM, compared with the control lymphoma cell lines derived from tumours with non-targeting control sgRNAs (\#219, \#220), as detected by both Western blotting (Fig. 5a) and intracellular flow cytometric analysis (Fig. 5b). We further noted that expression of the related pro-survival protein BCL-XL was highly variable across the individual lymphomas (Fig. 5a). Significantly, we found that all cell lines derived from $\mathrm{sg} B \mathrm{Cl}-2$ lymphomas were sensitized to venetoclax treatment (mean IC50 $=0.11 \mu \mathrm{M}$ ), which is in striking contrast to the standard lymphomas that arise in E $\mu$-Myctransgenic mice (mean IC50>1 $\mu \mathrm{M}$; Fig. 5c and Supplementary Fig. 4a, b). In addition, whilst the BCL-2 expressing lymphoma lines were less sensitive overall to treatment with the MCL-1 inhibitor 663845 than control EM-Myclymphoma lines, some of the BCL-2 expressing lines that displayed lower venetoclax sensitivity were still similarly sensitive to the MCL-1 inhibitor (Fig. $5 \mathrm{~d}$ and Supplementary Fig. 4c). 
These data confirm that we have been able to establish a novel model of aggressive B cell lymphoma, that is very similar to human DHL, i.e. double expression of C-MYC and BCL-2 and surface expression of CD19 ${ }^{18}$. Our results suggest that both the BCL-2 inhibitor venetoclax and MCL-1 inhibitors (already in clinical trials for $\mathrm{B}$ cell malignancies but not $\mathrm{DHL})^{33}$ could be used for the treatment of this disease in humans.

\section{Identification of venetoclax resistance factors in the new model of aggressive lymphoma using genome wide CRISPR activation screens.}

An important clinical issue is the emergence of resistance to venetoclax in patients on therapy. The generation of $E \mu-M y c / d C a s 9 a-S A M^{K I /+} / s g B c l-2$ lymphoma cell lines that are highly venetoclax sensitised provided a system in which whole genome CRISPR activation screens could be carried out to identify genes that confer drug resistance when upregulated. We transduced six replicates each of two venetoclax sensitive murine BCL-2 expressing lymphoma lines with a recently described mouse genome-wide sgRNA library. ${ }^{34}$ The cells were cultured for two weeks after transduction to permit induction of gene expression and were then subjected to treatment with vehicle (DMSO) or the indicated concentrations of venetoclax for a further two weeks (Fig. 6a). DNA samples were collected, and next generation sequencing was performed to identify the sgRNAs enriched in venetoclax-treated versus control cell populations. At all concentrations, venetoclax treatment led to a strong enrichment of a subset of sgRNAs compared to the DMSO treated control samples (Supplementary Fig. 5a). Notably, we found enrichment of sgRNAs upregulating two pro-survival BCL-2 family members, BCL-XL and MCL-1, that, based on current literature, would be expected to mediate resistance to venetoclax ${ }^{19}$ (Supplementary Fig. 5b). To our surprise, however, we found that sgRNAs targeting the underappreciated pro-survival BCL-2 family member A1 were the most dominant sgRNAs enriched by venetoclax treatment in both cell lines (Fig. 6b). This was particularly evident in IC80 doses of venetoclax, where multiple sgRNAs targeting A1 were highly significantly enriched (FDR < 0.05) compared to DMSO treated control groups (Supplementary Table 1 and Supplementary Data). To confirm that upregulation of A1 can confer protection from venetoclax induced killing, a sgRNA targeting the Bc/2a1a promoter was transduced into two $E \mu-M y c / d C a s 9 a-$ $S A M^{K l /+} / s g B C l-2$ cell lines. Upregulation of $A 1$ expression in these cells was confirmed by Western blotting (Fig. 6c). Cell competition assays in vitro confirmed that A1-activated cells possessed a striking survival advantage over parental $E \mu-M y c / d C a s 9 a-S A M^{K I /+} / s g B c l-2$ cells in the presence of venetoclax (Fig. 6d), confirming that upregulation of A1 confers resistance to venetoclax treatment. Similar results were obtained when cells were engineered to upregulate MCL-1 expression rather than A1 (Supplementary Fig. $5 c$, d). In addition to the genes encoding pro-survival BCL-2 pro-survival proteins, we also identified a number of other genes which may be interesting to investigate in the context of venetoclax resistance (Supplementary Fig. 5).

\section{Discussion}


Traditional CRISPR loss-of-function methodologies have become routine in medical research, permitting the identification and validation of new tumour suppressor genes and potential therapeutic targets which impact tumorigenesis or sensitivity to anti-cancer therapies when genetically perturbed. ${ }^{35}$ However, identification of oncogenic drivers relies on the enhanced expression of specific gene products and therefore requires different methodologies. While cDNA expression strategies have proven successful for some models, the expression levels achieved are often extreme, leading to non-physiological gene functions, and rarely are multiple transcript isoforms expressed as part of these experiments. ${ }^{9}$ In contrast, CRISPRa systems can induce gene expression from the endogenous genomic locus at physiologically or pathologically relevant levels. Of particular interest is the application of CRISPRa to generate faithful models of gene-driven disease which were previously unattainable due to these limitations. To enable this, we generated a CRISPRa mouse which can be used to activate gene expression in vivo and in vitro. This mouse expresses a dCas9-SAM cassette from the Rosa26 locus for robust expression across different cell types. We selected the SAM system because recent reports suggest that this system is capable of strong activity at multiple gene loci. ${ }^{36}$ Additionally, we employed the CAGG promoter because it has been shown to be amongst the most active polymerase II promoters across multiple tissues and cell lines. ${ }^{37}$ Using primary cells isolated from our CRISPRa mouse, we demonstrated induction of expression of B cell specific surface markers in T cells and vice versa. This demonstrates that even silenced genes can be induced with our gene activation model.

We have exploited our new and robust CRISPRa mouse model to establish a sought after pre-clinical model of aggressive lymphoma highly reminiscent of DHL. This disease is an aggressive form of DLBCL with currently limited treatment options. ${ }^{38}$ Thus far, there are no animal models that recapitulate this disease in any way. Although previous reports demonstrated a model of DHL by intercrossing $E \mu-M y C$ with $E \mu-B C l-2$ transgenic mice, ${ }^{16}$ these tumours were identified as immature haematopoietic progenitor tumours with no $\mathrm{Ig}$ or $\mathrm{Tcr}$ gene rearrangements and did not represent classical B cell lymphomas. Additionally, these tumours could not be grown in culture, which made it almost impossible to further characterize them or use them to identify new drug targets. Our BCL-2 expressing lymphomas are committed to the B cell lineage (Fig. 4d) and can readily be grown as cell lines, potentially due to their lower levels of $\mathrm{BCL}-2$ expression compared with the $E \mu-B C l-2 / E \mu-M y c$ model. While it is difficult to compare BCL-2 protein levels between the two models, the $E \mu-B C l-2 / E \mu-M y c$ double transgenic mice exhibited a much shorter tumour latency than our model (all mice showing severe disease by 40 days in the former vs 68 days for the model described here), indicating that BCL-2 expression was likely higher or switched on earlier in the $E \mu-B c l-2 / E \mu-M y c$ model.

The advantages of a murine model from which cell lines can be derived in vitro are the opportunities to increase our understanding of the disease and interrogate the efficacy of new therapeutic regimens. Most cell lines derived from our murine BCL-2 expressing lymphoma model showed a strong dependency on BCL-2 for their survival ${ }^{39}$ (mean IC50 $=0.11 \mu \mathrm{M}$ for treatment with the BCL-2 inhibitor venetoclax), which is in striking contrast to conventional $E \mu-M y c$ lymphoma cells which are almost exclusively dependent on MCL-1 for their survival. ${ }^{25,26}$ Interestingly though, our newly developed lymphoma cell lines also 
displayed sensitivity to MCL-1 inhibition, albeit at a reduced level compared to conventional $E \mu-M y C$ lymphomas. Of note, we identified one BCL-2 expressing lymphoma cell line which is fully resistant to MCL-1 inhibitor treatment, but still sensitive to venetoclax. Interestingly, human DHLs were expected to be highly venetoclax sensitive, however venetoclax monotherapy in patients with relapsed DLBCL, including a limited number of DHLs, resulted in low overall response rates ${ }^{40}$. However, these patients were heavily pre-treated and sample sizes were low, highlighting a need for more pre-clinical evidence that venetoclax therapy is worth pursuing for this disease. In this regard, it will be interesting to further understand how the change in survival factor dependency in some of our murine lymphomas developed. As most of the lymphoma cells showed sensitivity to either, or in many cases both, BCL-2 and MCL-1 inhibition, this finding also raises the possibility that combinations of BH3-mimetics targeting MCL-1 and BCL-2, perhaps with addition of further anti-cancer agents, could have a place in the treatment of patients with DHL. A similar regimen has recently been shown to be successful for the treatment of AML. ${ }^{41}$

Since recent reports have indicated emergence of resistant disease in patients with CLL and AML on venetoclax therapy ${ }^{42}$, we sought to exploit our venetoclax-sensitive murine lymphoma cell lines to identify potential resistance factors. We took advantage of the CRISPRa machinery already expressed in these cells to perform genome-wide activating library screens. Interestingly, we identified the pro-survival BCL-2 family member A1 (called BFL-1 in humans) as the dominant driver of venetoclax resistance in these cells. Our results expand upon the observation that BFL-1 expression correlates with upfront insensitivity to $\mathrm{BH} 3$ mimetics ${ }^{43}$ by showing for the first time in non biased CRISPR activation screens that acquired resistance to venetoclax can occur through $\mathrm{A} 1$ upregulation. A1 represents an exciting drug target, as deletion of $\mathrm{A} 1$ in murine models caused no overt abnormalities. ${ }^{44,45}$ Hence, a drug targeting A1/BFL- 1 is expected to cause no on-target toxicity to healthy cells and could likely be used in combination with venetoclax. Thus far, no direct inhibitors of $A 1$ exist, though CDK9 inhibitors have been reported to reduce A1 expression. ${ }^{43}$ However, CDK9 inhibitors also diminish the expression of MCL-1 and many other shortlived proteins; therefore on-target side effects are expected to be more substantial. Accordingly, clinical trials of BH3-mimetics targeting MCL-1 have recently led to some concerning side-effects. ${ }^{33}$ Our results suggest that $\mathrm{A} 1$ inhibition may represent an alternative to overcoming venetoclax resistance in the clinic.

In summary, we have developed a highly efficient CRISPR activation mouse model which can be readily adapted to address both basic biological and translationally-focused research questions across many disciplines.

\section{Methods}

\section{Construct cloning}

All single guide RNA (sgRNA) sequences used for the CRISPR activation system were designed using the sgRNA design tool developed by the Zhang Lab (https://portals.broadinstitute.org/gppx/crispick/public). sgRNA vectors (LV06, CRISPRa SAM U6-gRNA: EF1a-Puro) targeting the promoter region of the mouse 
Bcl-2 gene, Mdm2 gene and non-targeting control sgRNA vectors were purchased from Sigma-Aldrich. The puromycin resistance gene within the SAM sgRNA vector was replaced by blue fluorescent protein (BFP) coding sequences to generate a SAM sgRNA vector that expresses BFP (CRISPRa SAM U6-gRNA: EF1a-BFP). sgRNAs targeting the promoters of the mouse $C d 4, C d 19$ and Irf4 sgRNAs gene promoters were cloned into this vector. The sgRNA sequences are shown in Supplementary Table 2.

\section{Cell culture}

All cell lines were cultured at $37^{\circ} \mathrm{C}$ with $10 \% \mathrm{CO}_{2}$ in a humid atmosphere. The human embryonic kidney cell line expressing the SV40 large T antigen (293T) cell line was maintained in Dulbecco's modified Eagle's medium (DMEM, Gibco) containing 10\% (v/v) heat-inactivated Foetal Bovine Serum (HI-FBS; Sigma-Aldrich) and 100 U Penicillin-Streptomycin solution (Pen-Strep, Sigma-Aldrich).

The E $\mu$-Myc lymphoma-derived cell lines EMRK-1184 and MRE-721 were generated in our laboratory. The $E \mu-M y c / d C a s 9 a-S A M^{K I /+} / s g B c l-2$ (or sgMdm2, or non-targeting sgRNA) lymphoma cell lines were derived from tumour tissues of sick mice that had been lethally irradiated and then transplanted with $E \mu$ Myc/dCas9a-SAM ${ }^{K I /+}$ HSPCs that had been transduced with Bcl-2 (or Mdm2, or non-targeting control) sgRNAs. All E $\mu$-Myc lymphoma cell lines were cultured in DMEM, 10\% (v/v) HI-FBS, $23.8 \mathrm{mM}$ sodium bicarbonate (Merck), $1 \mathrm{mM}$ HEPES (Gibco), $13.5 \mu \mathrm{M}$ folic acid (Sigma-Aldrich), $0.24 \mathrm{mM}$ L-asparagine monohydrate (Sigma-Aldrich), $0.55 \mathrm{mM} \mathrm{L-arginine} \mathrm{monohydrochloride} \mathrm{(Sigma-Aldrich),} 22.2 \mathrm{mM} \mathrm{D-}$ glucose (Ajax), $100 \mathrm{U}-\mu \mathrm{g} / \mathrm{mL}$ Pen-Strep and $50 \mu \mathrm{M}$ 2-mercaptoethanol (2-ME, Sigma-Aldrich); this medium is subsequently referred to as FMA.

\section{Lentiviral production and target cell infection}

The lentiviral packaging system containing $5 \mu \mathrm{g}$ p-MDL, $2.5 \mu \mathrm{g}$ p-RSV-REV, $3 \mu \mathrm{g}$ p-VSVG and $10 \mu \mathrm{g}$ target DNA vector were transfected into $293 \mathrm{~T}$ cells using the calcium phosphate transfection method. ${ }^{46}$ Viral supernatants along with $8 \mu \mathrm{g} / \mathrm{mL}$ polybrene were added to $1 \times 10^{5} \mathrm{E \mu}-\mathrm{Myc}$ lymphoma cells and centrifuged at 2,200 rpm for $2 \mathrm{~h}$ at $32^{\circ} \mathrm{C}$. After aspiration of viral supernatants, target cells were resuspended in FMA medium and seeded onto 12-well plates.

For HSPC infection, the lentiviral packaging system containing $5 \mu \mathrm{g}$-MDL, $2.5 \mu \mathrm{g}$ p-RSV-REV, $5 \mu \mathrm{g}$ p-ECO envelope and $10 \mu \mathrm{g}$ target DNA vector were transfected into 293T cells using the calcium phosphate transfection method. ${ }^{46}$ Viruses were used to infect HSPCs derived from E14.5 foetal livers or activated B cell blasts or T cell blasts derived from splenocytes.

\section{Cell viability assay}

Cell viability assays were performed on $E \mu-M y c$ lymphoma cell lines to assess sensitivity to $\mathrm{BH} 3$ mimetic drug treatment. Cells were plated into 96 -well flat-bottom plates at a density of $3 \times 10^{4}$ cells per well and treated for $24 \mathrm{~h}$ with the MCL-1 inhibitor $\mathbf{5 3 8 4 5}$ (Active Biochem) or the BCL-2 inhibitor venetoclax (Active Biochem) at the indicated concentrations. Cell viability was determined by staining with $1 \mu \mathrm{g} / \mathrm{mL}$ 
propidium iodide followed by flow cytometric analysis using the LSR-II Analyzer (BD Biosciences). Flow cytometry data were analysed using FlowJo software.

\section{Haematopoietic analysis and flow cytometry}

Thymus, bone marrow, spleen and lymph node were harvested from dCas9a-SAM ${ }^{K / K I}$ mice. Single cell suspensions were prepared in PBS (Gibco) containing 5 mM EDTA (Merck) and 5\% (v/v) HI-FBS. Intracellular staining was performed using the Intracellular Fixation \& Permeabilization Buffer Set (eBioscience) according to the manufacturer's instructions. All fluorochrome conjugated antibodies used for extracellular or intracellular staining are listed in the Supplementary Table 3. Staining with PI (1 $\mu \mathrm{g} / \mathrm{mL}$ ) was used to exclude dead cells. Fluorescence was quantified using the LSR Fortessa X-20 Cell Analyzer (BD Biosciences) and data were analysed using FlowJo software (BD Biosciences).

\section{Haematopoietic reconstitution experiments}

The care and use of experimental animals were performed in accordance with the rules and guidelines set out by the WEHI Animal Ethics Committee. Female dCas9a-SAM ${ }^{K I / K I}$ mice were crossed with $E \mu$ $M y c^{T /+}$ male mice to produce $E \mu-M y c^{T /+} ; d C a s 9 A^{K I /+}$ embryos. The $E \mu-M y c / d C a s 9 a-S A M^{K I /+} H S P C s$ were isolated from foetal livers of E14.5 embryos and maintained in the foetal liver medium containing a-MEM GlutaMAX (Gibco), 10\% (v/v) HI-FBS, 100 U- $\mu \mathrm{g} / \mathrm{mL}$ Pen-Strep, 10 mM HEPES, 1 mM L-glutamine (Gibco), $1 \mathrm{mM}$ sodium pyruvate (Gibco), $50 \mu \mathrm{M}$ 2-ME, and supplemented with recombinant mouse cytokines made in house. The cytokines used for HSPC culture were $100 \mathrm{ng} / \mathrm{mL}$ mouse stem cell factor, $10 \mathrm{ng} / \mathrm{mL}$ mouse interleukin-2, $50 \mathrm{ng} / \mathrm{mL}$ mouse thrombopoietin and $10 \mathrm{ng} / \mathrm{mL}$ mouse fms-like tyrosine kinase 3 . All cytokines were produced and kindly provided by Dr. Jian-Guo Zhang (WEHI).

Supernatants containing ecotropically packaged lentiviruses expressing sgRNA were added onto retronectin-coated 12-well plates along with $8 \mu \mathrm{g} / \mathrm{mL}$ polybrene and centrifuged at 3,500 rpm for $90 \mathrm{~min}$ at $32^{\circ} \mathrm{C}$. After aspiration of viral supernatants, cultured HSPCs were seeded onto each well supplemented with $0.5 \mathrm{~mL}$ fresh medium + cytokines and incubated at $37^{\circ} \mathrm{C}$ overnight. The virus-infected HSPCs were then injected intravenously (i.v.) into lethally irradiated ( $2 \times 5.5$ Gy, $4 \mathrm{~h}$ apart) C57BL/6-Ly5.1 recipient mice. Reconstituted mice were monitored for lymphoma development and overall appearance. Survival time was defined as the time from transplantation until reconstituted mice had to be sacrificed due to ethical reasons. Peripheral blood and tumour tissues collected from mice at the ethical endpoint were used for Western blotting, generation of cell lines and flow cytometric analysis.

\section{Culture and infection of primary immune cells}

Naïve B cells were cultured in RPMI 1640 medium supplemented with 10\% (v/v) HI-FBS, $100 \mathrm{U}-\mu \mathrm{g} / \mathrm{mL}$ Pen-Strep, 1\% L-glutamine, 1\% non-essential amino acids (Gibco), 1\% HEPES, $1 \%$ sodium pyruvate and 50 $\mu \mathrm{M}$ 2-ME. T cells were cultured in FMA medium plus $10 \mathrm{ng} / \mathrm{mL}$ mouse interleukin-2. Naïve B cells and T cells were stimulated with $10 \mu \mathrm{g} / \mathrm{mL}$ LPS (B cells) or $2 \mu \mathrm{g} / \mathrm{mL}$ concanavalin A plus $10 \mathrm{ng} / \mathrm{mL}$ mouse interleukin-2 (T cells). 
For lentiviral infection, $5 \times 10^{5}$ activated $B$ cells per well were added onto retronectin-coated 12-well plates. Lentiviruses expressing sgRNA along with $8 \mu \mathrm{g} / \mathrm{mL}$ polybrene were added onto cells and centrifuged at $1,200 \mathrm{rpm}$ for $90 \mathrm{~min}$ at $28^{\circ} \mathrm{C}$. After aspiration of viral supernatants, fresh medium was added into each well and cells were incubated under normal conditions. Lentiviral supernatants along with $8 \mu \mathrm{g} / \mathrm{mL}$ polybrene were added to $1 \times 10^{6}$ activated T cells and centrifuged at 2,200 rpm for $2 \mathrm{~h}$ at $32^{\circ} \mathrm{C}$. After aspiration of viral supernatants, $\mathrm{T}$ cells were resuspended in fresh medium and seeded onto 12-well plates.

\section{Western blotting}

Cell lysates were prepared in RIPA lysis buffer (50 mM Tris-HCl (pH 8.0), $150 \mathrm{mM} \mathrm{NaCl}, 1 \%$ NP-40, 0.5\% Sodium Deoxycholate, $0.1 \%$ SDS) in the presence of EDTA-free protease inhibitor tablets (Roche) and incubated on ice for $15 \mathrm{~min}$. Protein concentrations were measured using the BCA Protein Assay Kit (Thermo Fisher) following the manufacturer's protocols. A total of $15 \mu \mathrm{g}$ protein samples were loaded onto NuPAGE 4\%-12\% Bis-Tris $1.5 \mathrm{~mm}$ gels (Life Technologies) and proteins were size fractionated by gel electrophoresis. The iBlot 2 Dry Blotting System (Life Technologies) was used to transfer proteins onto $0.2 \mathrm{~mm}$ nitrocellulose membranes. Membranes were blocked in $5 \%(\mathrm{~m} / \mathrm{v})$ skim milk and incubated with primary antibodies overnight at $4^{\circ} \mathrm{C}$. Primary antibodies used for Western blotting are listed in Supplementary Table 4. Membranes were washed and incubated with appropriate horseradish peroxidase (HRP)-conjugated secondary antibodies to detect mouse, rat or rabbit lgG (Southern Biotech). Antibody bound proteins on membranes were visualised by adding the Luminata Forte Western HRP substrate (Millipore) and imaging was performed using the ChemiDoc XRS + machine (Bio-Rad).

\section{CRISPR screen}

Whole genome CRISPR/Cas9 activating screens were performed in $E \mu-M y c / d C a s 9 a-S A M^{K I /+} / s g B c l-2$ lymphoma-derived cell lines. To prepare sgRNA library virus, $10 \mu \mathrm{g}$ Caprano CRISPRa sgRNA library (A and $B$ combined) ${ }^{34}$ was transfected into 293 T cells along with $5 \mu \mathrm{g} \mathrm{p}$-VSVG and $10 \mu \mathrm{g}$ psPAX2 as described above. E $\mu$-Myc/dCas9a-SAM ${ }^{K I /+} / s g B c l-2$ lymphoma-derived cell lines (\#214, \#216), which are highly sensitive to venetoclax, were split into 6 replicates of $3 \times 10^{5}$ cells and infected as described above for the $E \mu$-Myc lymphoma cells. Infected cells were expanded in culture for 2 weeks. For each replicate infection, $7 \times 10^{6}$ cells were transferred into three T75 flasks and treated with either DMSO (negative control), or IC50 doses of venetoclax (214: $10 \mathrm{nM}, 216: 5 \mathrm{nM})$, or IC80 (214: $30 \mathrm{nM}, 216: 15 \mathrm{nM})$. Cells were passaged every $3-5$ days as they became confluent and fresh drug was added. Cells receiving the IC50 dose of venetoclax were split into two new T75 flasks after 4 days of treatment. One culture was maintained at an IC50 dose while the dose for the other was ramped up slowly over time until an IC100 dose (214: $100 \mathrm{nM}, 216: 50 \mathrm{nM}$ ) was reached. After a total of 14 days of drug treatment, pellets of $2 \times 10^{6}$ cells were collected for all treatments. DNA was extracted using a DNeasy Blood \& Tissue Kit (QIAGEN). sgRNAs were amplified from $100 \mathrm{ng}$ of DNA using GoTaq Green Master Mix (Promega) according to the manufacturer's protocol. The following primers were used, which had been modified with unique overhangs to create a set of indexing primers for Illumina sequencing: FWD: 3 '- 
TGCTTACCGTAACTTGAAAGTA - 5' and REV: 5'-AATACGAGCAGACCCTGATG-3'. PCRs were performed in triplicate for each sample. Products were pooled, cleaned up using Ampure XP beads (Beckman Coulter) and sequenced on an Illumina NextSEq. For each sample, the number of reads mapping to each sgRNA in the library (as a proportion of the total number of reads for that sample) was calculated. For bar graphs, sgRNAs which made up at least $10 \%$ of the total sgRNAs detected for each sample were plotted.

\section{Validation of hits from the CRISPR screens}

To validate hits identified from CRISPR screens, select sgRNA sequences from the Caprano library were individually cloned into pXPR_502. Virus was prepared and used to transduce $1 \times 10^{5} \mathrm{E} \mu-\mathrm{Myc} / \mathrm{dCas} 9 \mathrm{a}$ $S A M^{K I /+} / s g B c l-2$ lymphoma-derived cells as described above. Infected cells were cultured for 2-3 weeks to allow time for gene induction. Meanwhile, parental $E \mu-M y c / d C a s 9 a-S A M^{K I /+} / s g B c l-2$ lymphomaderived cell lines were transduced with a retroviral vector expressing high GFP (pMIG) as described above, using $10 \mu \mathrm{g}$ pMIG, $5 \mu \mathrm{g}$ GAG and $5 \mu \mathrm{g}$ ENV. GFP-high cells were sorted on a FACSAria III flow cytometer (BD Biosciences). GFP-high parental cells were mixed 1:1 with cells carrying the sgRNAs for validation. Mixed cultures were treated with DMSO (negative control) or an IC50 dose of venetoclax for up to 2 weeks. Cultures were passaged every 2-3 days, fresh drug added, and the proportions of each cell population monitored by flow cytometry.

\section{Statistical analysis}

All data were analysed by Prism software (Version 8.2.0, GraphPad Software). The comparison between two groups was determined by student's $t$-test. For more than two groups, multiple $t$-tests or two-way ANOVA were performed to evaluate differences between the groups. Kaplan-Meier survival curves were plotted to represent the viability of HSPC reconstituted mice. Data are represented as means \pm standard error of the mean (SEM) and significance between groups are measured by $P$ value that is statistically significant as $*=P<0.05, * *=P<0.01$ and $n s=$ no significant difference.

Differential enrichment analyses were conducted using edgeR software package edgeR v3.30.3. 47,48 sgRNAs were filtered by using the method described ${ }^{49}$ and library sizes were normalised using upperquartile method. ${ }^{50}$ Counts were then transformed to log2 (counts per million) with associated precision weights, using the voom method. ${ }^{51}$ Differential enrichment between treatments was assessed using empirical Bayes moderated t-statistics. ${ }^{52}$ The $P$ values were adjusted to control the false discovery rate (FDR) using the method of Benjamini and Hochberg. ${ }^{53}$

\section{References}

1. Pickar-Oliver A, Gersbach CA. The next generation of CRISPR-Cas technologies and applications. Nat Rev Mol Cell Biol 20, 490-507 (2019).

2. Aoyama T, Chua NH. A glucocorticoid-mediated transcriptional induction system in transgenic plants. Plant J 11, 605-612 (1997). 
3. Maeder ML, Linder SJ, Cascio VM, Fu Y, Ho QH, Joung JK. CRISPR RNA-guided activation of endogenous human genes. Nat Methods 10, 977-979 (2013).

4. Mali P, et al. CAS9 transcriptional activators for target specificity screening and paired nickases for cooperative genome engineering. Nat Biotechno/ 31, 833-838 (2013).

5. Perez-Pinera P, et al. RNA-guided gene activation by CRISPR-Cas9-based transcription factors. Nature Methods 10, 973-976 (2013).

6. Gilbert LA, et al. CRISPR-mediated modular RNA-guided regulation of transcription in eukaryotes. Cell 154, 442-451 (2013).

7. Chavez A, et al. Highly efficient Cas9-mediated transcriptional programming. Nat Methods 12, 326328 (2015).

8. Gilbert LA, et al. Genome-Scale CRISPR-Mediated Control of Gene Repression and Activation. Cell 159, 647-661 (2014).

9. Konermann $\mathrm{S}$, et al. Genome-scale transcriptional activation by an engineered CRISPR-Cas9 complex. Nature 517, 583-588 (2015).

10. Tanenbaum Marvin E, Gilbert Luke A, Qi Lei S, Weissman Jonathan S, Vale Ronald D. A ProteinTagging System for Signal Amplification in Gene Expression and Fluorescence Imaging. Cel/ 159, 635-646 (2014).

11. Ventura A, Dow LE. Modeling Cancer in the CRISPR Era. Annual Review of Cancer Biology 2, 111131 (2018).

12. Hanahan D, Weinberg RA. The hallmarks of cancer. Cell 100, 57-70 (2000).

13. Potts MA, McDonald JA, Sutherland KD, Herold MJ. Critical cancer vulnerabilities identified by unbiased CRISPR/Cas9 screens inform on efficient cancer Immunotherapy. Eur J Immuno/ 50, 18711884 (2020).

14. Aukema SM, et al. Double-hit B-cell lymphomas. Blood 117, 2319-2331 (2011).

15. Johnson NA, et al. Lymphomas with concurrent BCL2 and MYC translocations: the critical factors associated with survival. Blood 114, 2273-2279 (2009).

16. Strasser A, Harris AW, Bath ML, Cory S. Novel primitive lymphoid tumours induced in transgenic mice by cooperation between myc and bcl-2. Nature 348, 331-333 (1990).

17. Ravà $\mathrm{M}$, et al. Therapeutic synergy between tigecycline and venetoclax in a preclinical model of MYC/BCL2 double-hit B cell lymphoma. Sci Trans/ Med 10, (2018).

18. Chen B-J, Fend F, Campo E, Quintanilla-Martinez L. Aggressive B-cell lymphomas-from morphology to molecular pathogenesis. Annals of Lymphoma 3, (2019).

19. Merino D, Kelly GL, Lessene G, Wei AH, Roberts AW, Strasser A. BH3-Mimetic Drugs: Blazing the Trail for New Cancer Medicines. Cancer Cel/ 34, 879-891 (2018).

20. Guièze R, et al. Mitochondrial Reprogramming Underlies Resistance to BCL-2 Inhibition in Lymphoid Malignancies. Cancer Cell 36, 369-384.e313 (2019). 
21. Thijssen $\mathrm{R}$, et al. Intact TP-53 function is essential for sustaining durable responses to BH3-mimetic drugs in leukemias. Blood 137, 2721-2735 (2021).

22. Lois C, Hong EJ, Pease S, Brown EJ, Baltimore D. Germline transmission and tissue-specific expression of transgenes delivered by lentiviral vectors. Science 295, 868-872 (2002).

23. Adams JM, et al. The c-myc oncogene driven by immunoglobulin enhancers induces lymphoid malignancy in transgenic mice. Nature 318, 533-538 (1985).

24. Aubrey BJ, et al. An inducible lentiviral guide RNA platform enables the identification of tumoressential genes and tumor-promoting mutations in vivo. Cell Rep 10, 1422-1432 (2015).

25. Kelly GL, et al. Targeting of MCL-1 kills MYC-driven mouse and human lymphomas even when they bear mutations in p53. Genes Dev 28, 58-70 (2014).

26. Kotschy A, et al. The MCL1 inhibitor 563845 is tolerable and effective in diverse cancer models. Nature 538, 477-482 (2016).

27. Thai TH, et al. Regulation of the germinal center response by microRNA-155. Science 316, 604-608 (2007).

28. Low MSY, et al. IRF4 Activity Is Required in Established Plasma Cells to Regulate Gene Transcription and Mitochondrial Homeostasis. Cell Rep 29, 2634-2645.e2635 (2019).

29. Michalak EM, et al. Puma and to a lesser extent Noxa are suppressors of Myc-induced lymphomagenesis. Cell Death Differ 16, 684-696 (2009).

30. Haupt Y, Maya R, Kazaz A, Oren M. Mdm2 promotes the rapid degradation of p53. Nature 387, 296299 (1997).

31. Vassilev LT, et al. In vivo activation of the p53 pathway by small-molecule antagonists of MDM2. Science 303, 844-848 (2004).

32. Strasser A, Harris AW, Huang DC, Krammer PH, Cory S. Bcl-2 and Fas/APO-1 regulate distinct pathways to lymphocyte apoptosis. Embo j 14, 6136-6147 (1995).

33. Bolomsky A, et al. MCL-1 inhibitors, fast-lane development of a new class of anti-cancer agents. $J$ Hematol Oncol 13, 173 (2020).

34. Sanson KR, et al. Optimized libraries for CRISPR-Cas9 genetic screens with multiple modalities. Nat Commun 9, 5416 (2018).

35. Sánchez-Rivera FJ, Jacks T. Applications of the CRISPR-Cas9 system in cancer biology. Nat Rev Cancer 15, 387-395 (2015).

36. Chavez A, et al. Comparison of Cas9 activators in multiple species. Nat Methods 13, 563-567 (2016).

37. Qin JY, et al. Systematic comparison of constitutive promoters and the doxycycline-inducible promoter. PLoS One 5, e10611 (2010).

38. Friedberg JW. How I treat double-hit lymphoma. Blood 130, 590-596 (2017).

39. Uchida A, et al. Targeting BCL2 with venetoclax is a promising therapeutic strategy for "doubleproteinexpression" Iymphoma with MYC and BCL2 rearrangements. Haematologica 104, 1417-1421 
(2019).

40. Davids MS, et al. Phase I First-in-Human Study of Venetoclax in Patients With Relapsed or Refractory Non-Hodgkin Lymphoma. J Clin Onco/35, 826-833 (2017).

41. Moujalled DM, et al. Combining BH3-mimetics to target both BCL-2 and MCL1 has potent activity in pre-clinical models of acute myeloid leukemia. Leukemia 33, 905-917 (2019).

42. Blombery P, et al. Acquisition of the Recurrent Gly101Val Mutation in BCL2 Confers Resistance to Venetoclax in Patients with Progressive Chronic Lymphocytic Leukemia. Cancer Discov 9, 342-353 (2019).

43. Boiko S, et al. Targeting Bfl-1 via acute CDK9 inhibition overcomes intrinsic BH3-mimetic resistance in lymphomas. Blood 137, 2947-2957 (2021).

44. Schenk RL, et al. Characterisation of mice lacking all functional isoforms of the pro-survival BCL-2 family member A1 reveals minor defects in the haematopoietic compartment. Cell Death Differ 24, 534-545 (2017).

45. Tuzlak S, et al. The BCL-2 pro-survival protein A1 is dispensable for T cell homeostasis on viral infection. Cell Death Differ 24, 523-533 (2017).

46. Herold MJ, van den Brandt J, Seibler J, Reichardt HM. Inducible and reversible gene silencing by stable integration of an shRNA-encoding lentivirus in transgenic rats. Proc Natl Acad Sci U S A 105, 18507-18512 (2008).

47. Dai $Z$, et al. edgeR: a versatile tool for the analysis of shRNA-seq and CRISPR-Cas9 genetic screens. F1000Res 3, 95 (2014).

48. McCarthy DJ, Chen Y, Smyth GK. Differential expression analysis of multifactor RNA-Seq experiments with respect to biological variation. Nucleic Acids Res 40, 4288-4297 (2012).

49. Chen Y, Lun AT, Smyth GK. From reads to genes to pathways: differential expression analysis of RNASeq experiments using Rsubread and the edgeR quasi-likelihood pipeline. F1000Res 5, 1438 (2016).

50. Bullard JH, Purdom E, Hansen KD, Dudoit S. Evaluation of statistical methods for normalization and differential expression in mRNA-Seq experiments. BMC Bioinformatics 11, 94 (2010).

51. Law CW, Chen Y, Shi W, Smyth GK. voom: Precision weights unlock linear model analysis tools for RNA-seq read counts. Genome Biol 15, R29 (2014).

52. Smyth GK. Linear models and empirical bayes methods for assessing differential expression in microarray experiments. Stat Appl Genet Mol Bio/3, Article3 (2004).

53. Benjamini Y, Hochberg Y. Controlling the False Discovery Rate: A Practical and Powerful Approach to Multiple Testing. Journal of the Royal Statistical Society: Series B (Methodological) 57, 289-300 (1995).

\section{Declarations}

\section{Acknowledgements}


We thank C Gatt,C Epifanio and G Siciliano for expert animal care; B Helbert and K Mackwell for genotyping; S Monard and his team for help with flow cytometry; MA Anderson and D Hilton for insightful discussions and all the members of the Herold and Kelly laboratories. This work was supported by grants and fellowships from the Australian National Health and Medical Research Council (NHMRC) (Project Grants 1159658,1186575 and 1145728 to MJH, 1143105 to MJH and AS, Ideas Grants 2002618 and 2001201 to GLK, Program Grant 1113133 to AS and Fellowships 1020363 to AS, 1156095 to MJH), the Leukemia and Lymphoma Society of America (LLS SCOR 7015-18 to AS, GLK, MJH), the Cancer Council of Victoria (project grant 1147328 and 2021 Grant In Aid to MJH, 1052309 to AS, 1147328 to GLK and Venture Grant to MJH and AS), Victorian Cancer Agency (MCRF Fellowship 17028 to GLK, Leukaemia Foundation of Australia (grant to AS and GLK), Phenomics Australia (to AJK and MJH) the estate of Anthony (Toni) Redstone OAM (AS and GLK), the Craig Perkins Cancer Research Foundation (GLK), the Dyson Bequest (GLK), the Harry Secomb Trust (GLK) as well as by operational infrastructure grants through the Australian Government Independent Research Institute Infrastructure Support Scheme (361646 and 9000220) and the Victorian State Government Operational Infrastructure Support Program.

\section{Authorship Contributions}

YD, STD performed and supervised all experiments, MAP, AJK, MP, SW, LT, GG contributed some of the experiments, AH, AS, BH, QZ gave critical advice, GLK and MJH designed and supervised all experiments. All authors were involved in writing the manuscript.

\section{Competing interests}

The authors have no competing interests to declare.

\section{Figures}



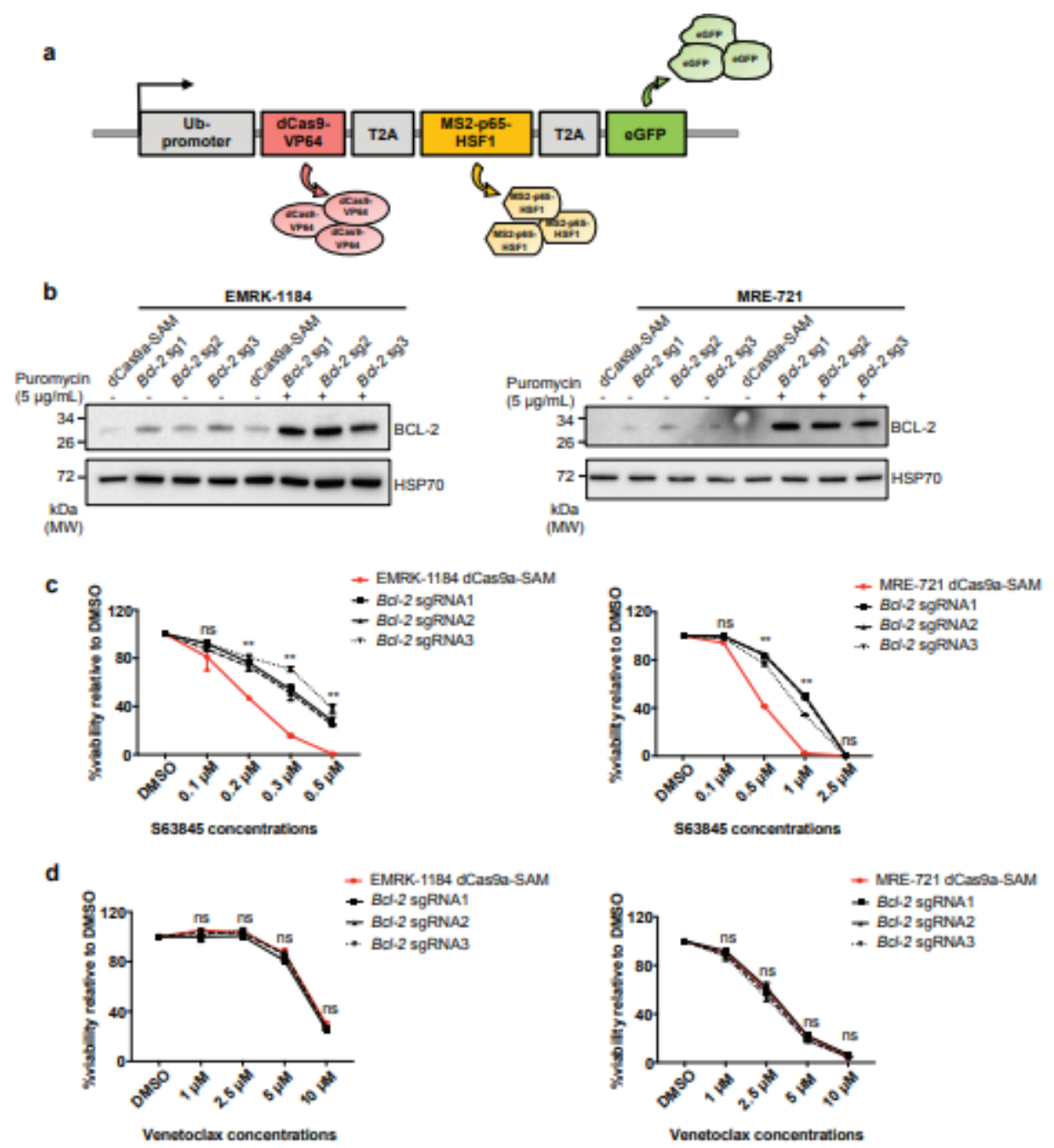

\section{Figure 1}

Generation and Validation of pdCas9a-SAM construct in E $\mu$-Myc lymphoma cell lines. a Schematic representation of the pdCas9a-SAM lentiviral construct. b Western blot analysis for BCL-2 levels in

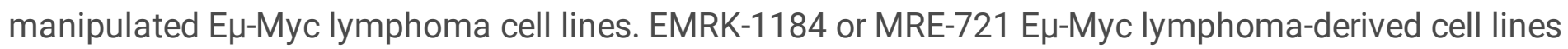
were transduced with pdCas9a-SAM only or pdCas9a-SAM plus Bcl-2 sgRNAs. Cell lysates were harvested from transduced cell lines, before or after puromycin-selection and expression of the indicated proteins were examined by Western blotting. Probing for the heat shock protein 70 (HSP70) served as a

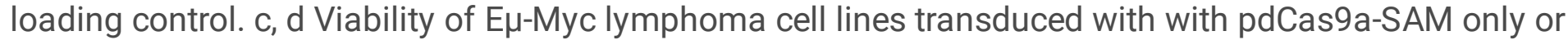
pdCas9a-SAM plus Bcl-2 sgRNAs. Cells were treated with the MCL-1 inhibitor S63845 or the BCL-2 inhibitor venetoclax at the indicated drug concentrations. Cell viability was determined at $24 \mathrm{~h}$ by propidium iodide $(\mathrm{PI})$ staining and subsequent flow cytometric analysis. Data are represented as mean \pm $\mathrm{SEM}, \mathrm{n}=3$ independent experiments. The statistical significance was determined using student's t-test. $\mathrm{ns}=$ no significant difference, ${ }^{*}=\mathrm{P}<0.01$. 
a

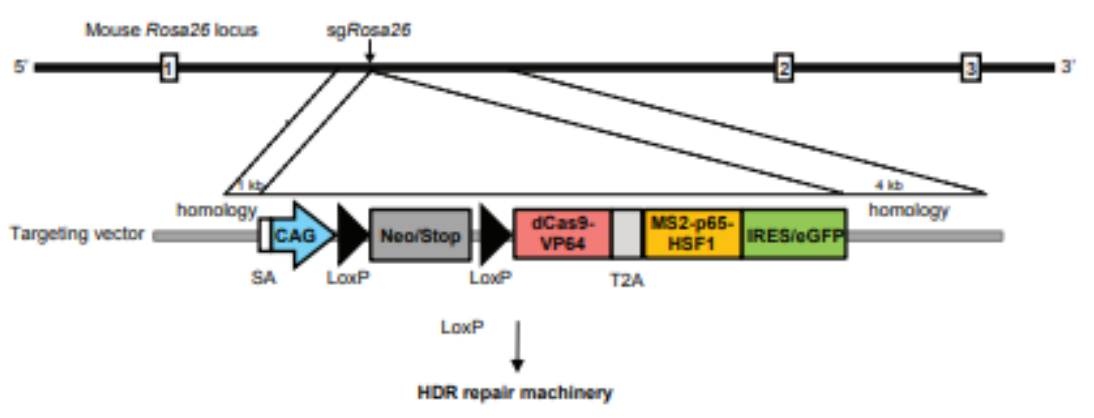

b

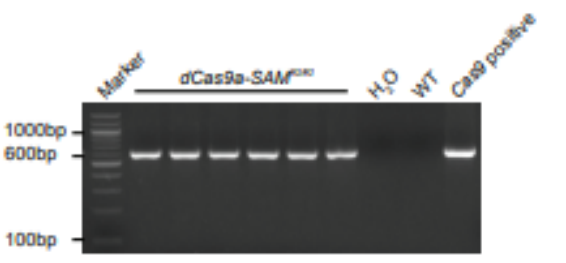

c

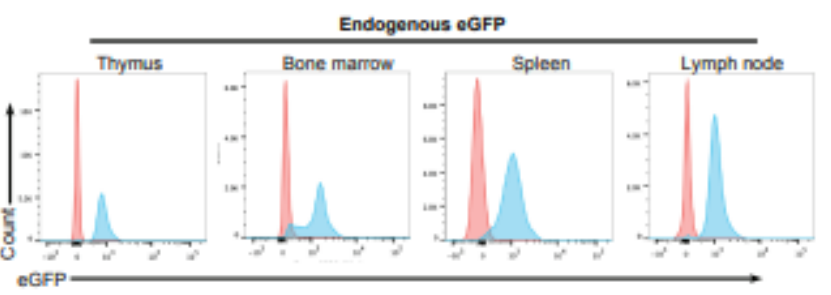

d

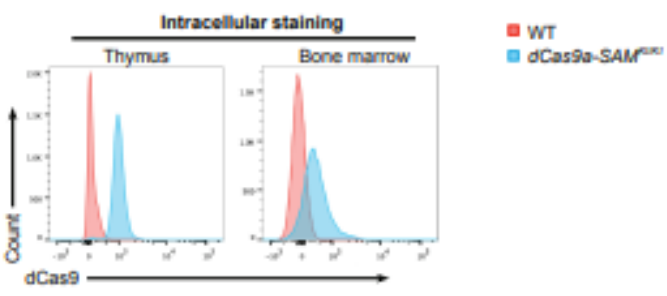

Figure 2

Establishment and validation of dCas9a-SAMKI/KI mice. a Strategy for targeting of the dCas9a-SAM cassette into the mouse Rosa26 locus. Rosa26 sgRNA guides the Cas 9 nuclease to introduce doublestrand breaks at a specific target site in the Rosa26 locus. Repair of the cut by endogenous homologydirected repair (HDR) mechanism using homology sequences present in the pRosa26-dCas9a-SAM vector enables the insertion of the dCas9a-SAM cassette into the Rosa26 locus. b PCR based validation of dCas9 expression in dCas9a-SAMKI/KI mice. Desired bands run at a size of $621 \mathrm{bp}$. c, d Representative flow cytometric data on eGFP expression or intracellular dCas9 staining in the indicated haematopoietic tissues derived from wildtype (WT; negative control) or dCas9a-SAMKI/KI mice. 

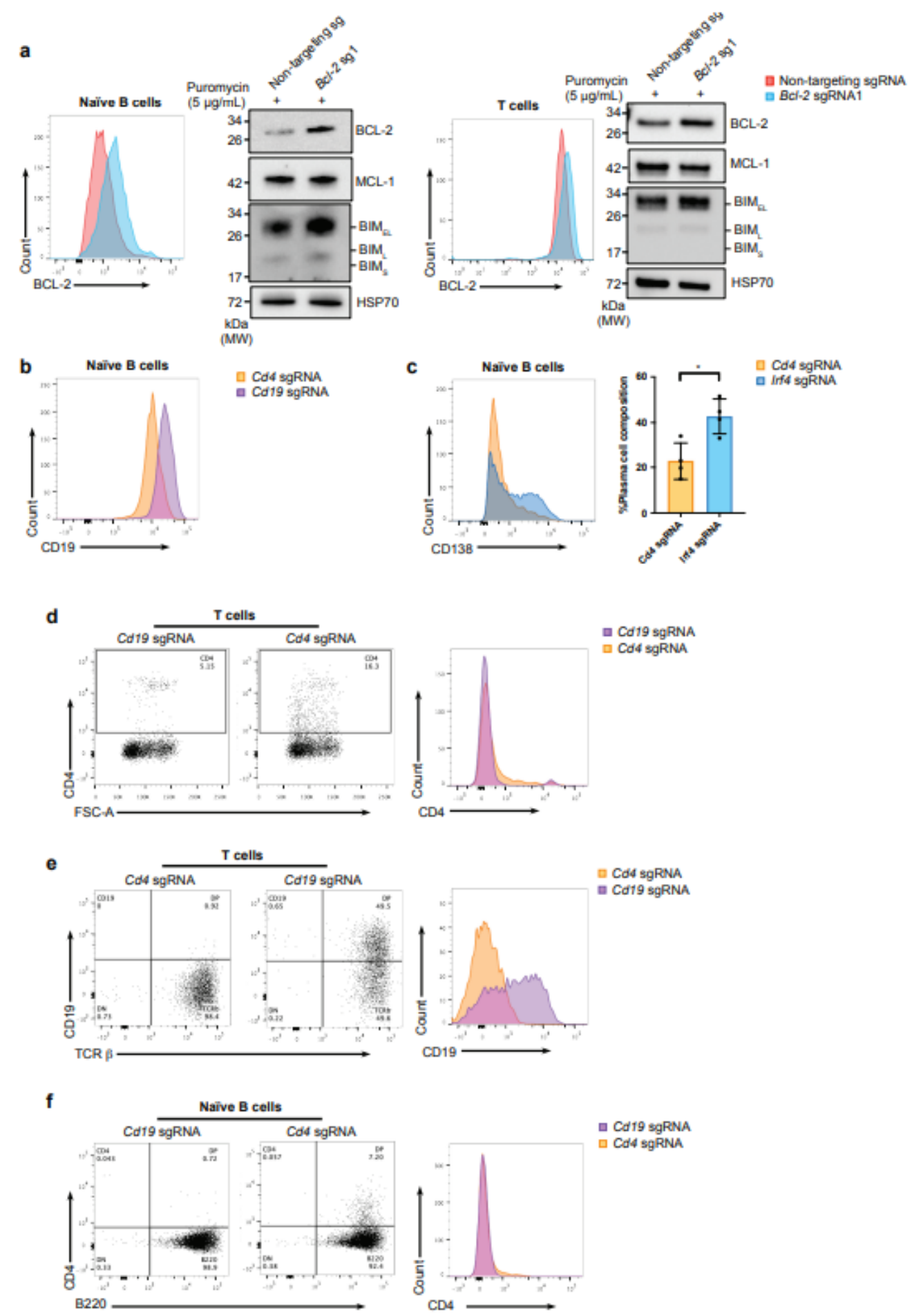

\section{Figure 3}

Induction of robust gene expression in primary murine B cells and T cells. Naïve B cells or T cells were isolated from the dCas9a-SAMKI/KI mice and cultured in vitro in medium containing LPS or ConA plus IL2, respectively. a Mitogen activated B or T cells were transduced with the Bcl-2 sgRNA1 and selected with $5 \mu \mathrm{g} / \mathrm{mL}$ puromycin for 2 days. Robust BCL-2 expression was validated by intracellular staining for BCL-2 protein and Western blot analysis. Probing for HSP70 served as a loading control on Western blots. 
b, c Activated B cells were transduced with Cd19 or Irf4 sgRNAs. The expression of CD19 or CD138 (impacted by IRF4 expression) was analysed by flow cytometry. Cells transduced with Cd4 sgRNAs served as the negative control. Data are presented as mean \pm SEM. The statistical significance was determined by the student's t-test. * $=P<0.05$. $d$, e T cell blast were transduced with $\mathrm{Cd} 4$ or $\mathrm{Cd} 19$ sgRNAs. T cells were gated on TCR $\beta+$. The expression of CD4 or CD19 was analysed by flow cytometry. Cells transduced with $\mathrm{Cd} 19$ or $\mathrm{Cd} 4 \mathrm{sgRNAs}$ served as negative controls. $\mathrm{f}$ Activated $\mathrm{B}$ cells were transduced with Cd4 sgRNAs. B cells were gated on B220+. The expression of CD4 was analysed by flow cytometry. Cells transduced with Cd19 sgRNA served as the negative control.

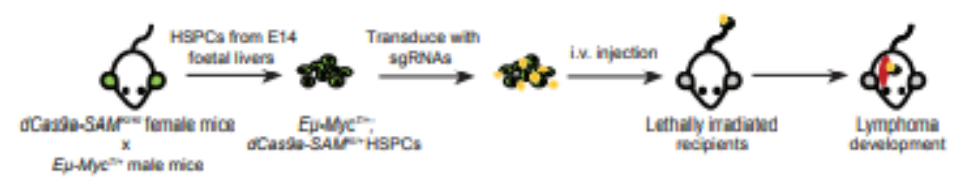

b
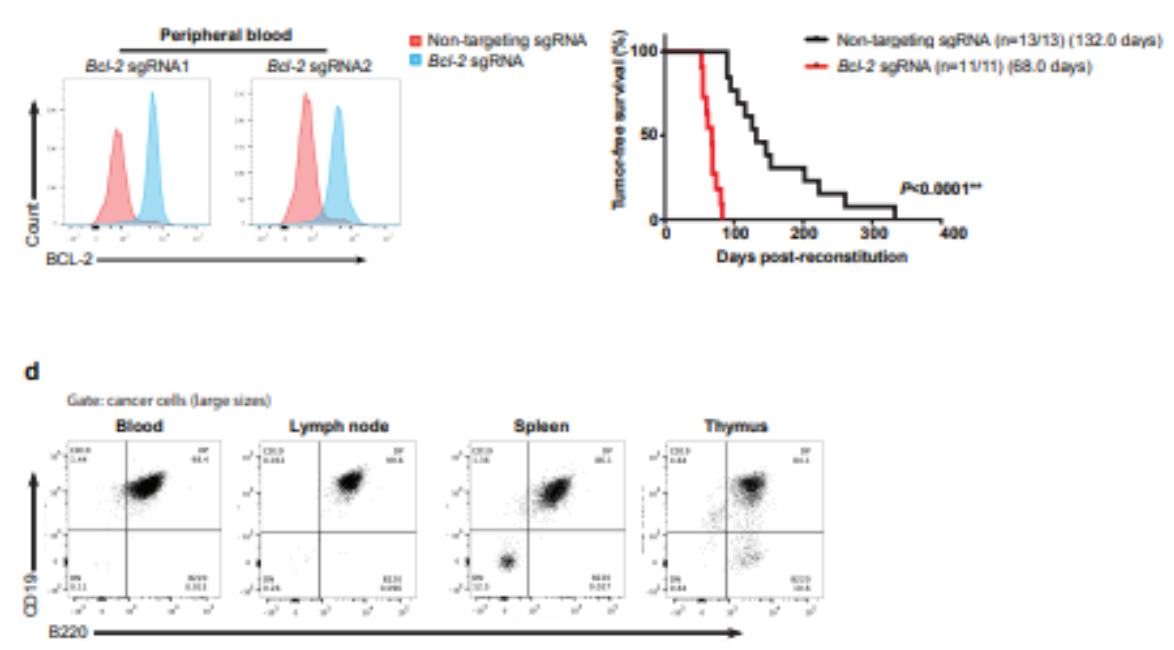

。
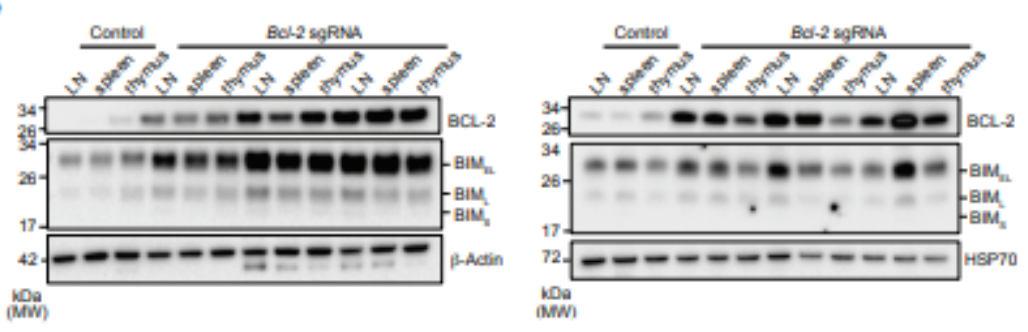

\section{Figure 4}

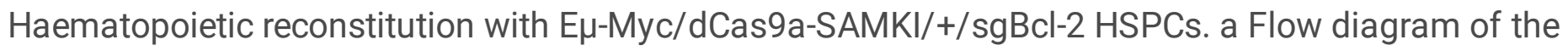
haematopoietic reconstitution assay. Female dCas9a-SAMKI/KI mice were crossed with male E $\mu-M y c T /+$

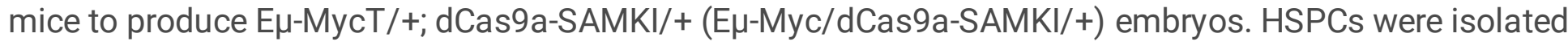
from the foetal livers of E14.5 embryos and cultured in vitro for sgRNA transduction. HSPCs were transduced with sgRNAs (sgBcl-2 or non-targeting control sgRNAs) and then injected into lethally irradiated recipient mice by intravenous (i.v.) injection. Enlarged spleen, lymph nodes or thymus, and a high level of white blood cells were considered as signs of malignant disease to identify lymphoma- 
bearing mice. $\mathrm{b}$ The expression of BCL-2 in cells from the peripheral blood of reconstituted mice was determined by intracellular staining and flow cytometry. c Kaplan-Meier survival curve of reconstituted mice transplanted with E $\mu$-Myc/dCas9a-SAMKI/+/sgBcl-2 HSPCs or non-targeting controls. $n / n=$ numbers of sick mice/numbers of total recipient mice. Data are combined of two independent reconstitution experiments and $P$ value is as indicated. ${ }^{*}=P<0.01$. $d$ Characterisation of E $\mu$ $\mathrm{Myc} / \mathrm{dCas} 9 \mathrm{a}-\mathrm{SAMKI} /+$ /sgBcl-2 lymphoma phenotype. Blood, lymph nodes, spleen and thymus were harvested from sick mice for staining and flow cytometric analysis. Tumour cells were gated on a larger size than normal haematopoietic cells. e The expression of BCL-2 and BIM in tumour tissues of mice

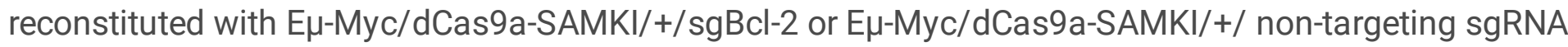
HSPCs is shown by Western blotting. Probing for $\beta$-actin or HSP70 served as loading controls. 6 individual sgBcl-2 reconstituted mice and 2 individual control mice were used for Western blot analysis.

a
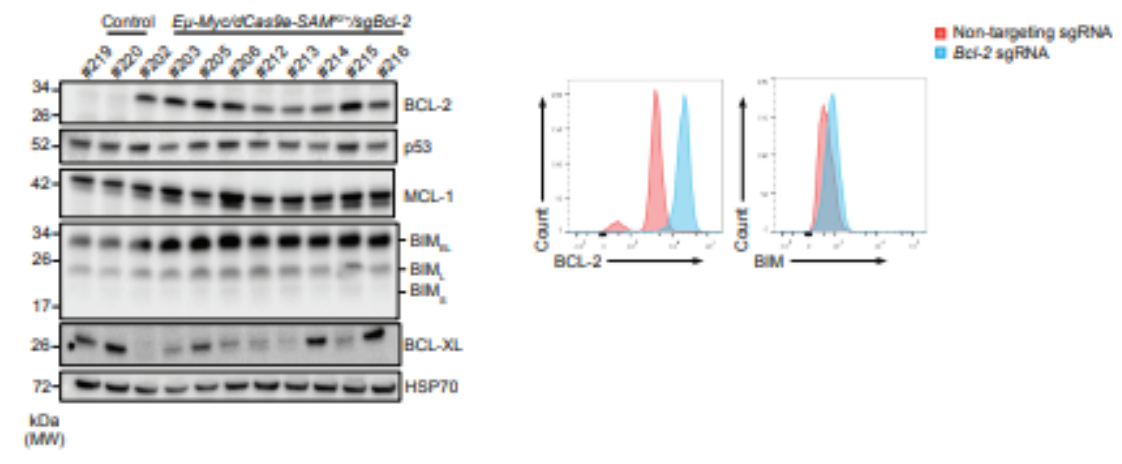

c

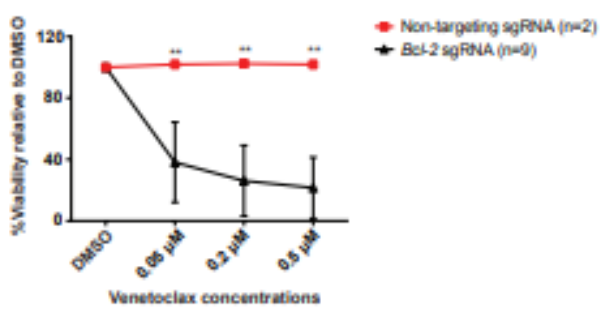

d

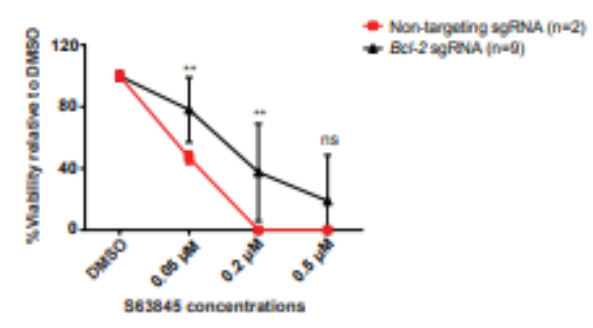

Figure 5

Characterisation of $\mathrm{E} \mu-\mathrm{Myc} / \mathrm{dCas} 9 \mathrm{a}-\mathrm{SAMKI} /+/ \mathrm{sgBcl}-2$ lymphoma-derived cell lines. a The expression of the indicated proteins in control lymphoma-derived cell lines or E $\mu-M y c / d C a s 9 a-S A M K I /+/ s g B c l-2$ lymphoma-derived cell lines were determined by Western blot analysis. The indicated numbers of cell lines represent the animal numbers of sick mice from which the cell lines had been derived. Probing for 
HSP70 served as a loading control. b The expression of BCL-2 and BIM in cell lines was determined by intracellular staining and flow cytometry. c, d Cell viability upon treatment with the BCL-2 inhibitor venetoclax or the MCL-1 inhibitor S63845. E $4-M y c / d C a s 9 a-S A M K I /+/ s g B c l-2$ lymphoma cell lines $(n=9)$ and control cell lines $(n=2)$ were treated for $24 \mathrm{~h}$ with the indicated concentrations of venetoclax or S63845. Cell viability was determined by propidium iodide $(\mathrm{PI})$ staining followed by flow cytometric analysis. Data are presented as mean \pm SEM and the statistical significance was evaluated by student's $t-$ test as ** $=\mathrm{P}<0.01$.

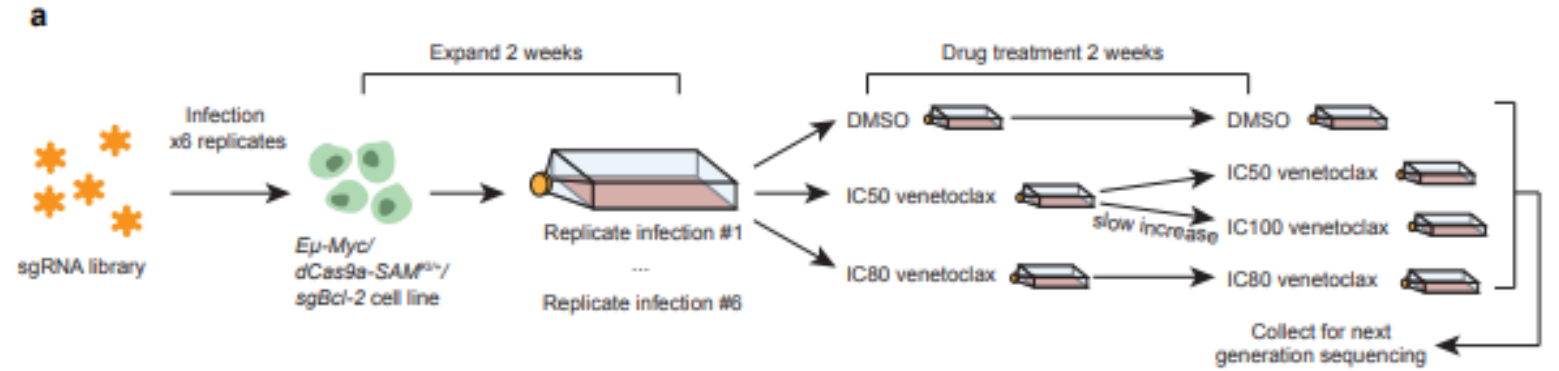

b

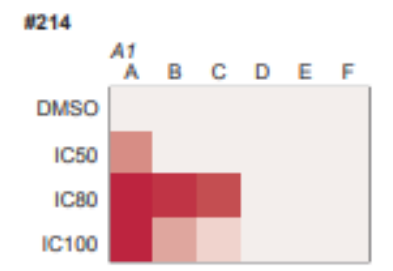

w216

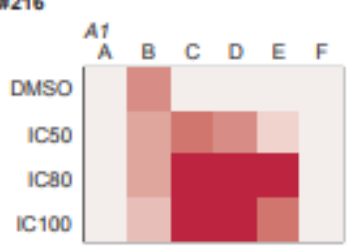

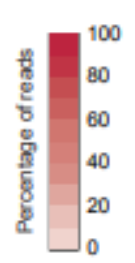

c

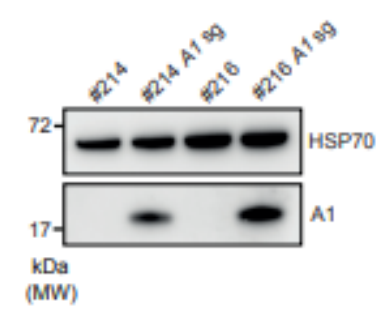

d
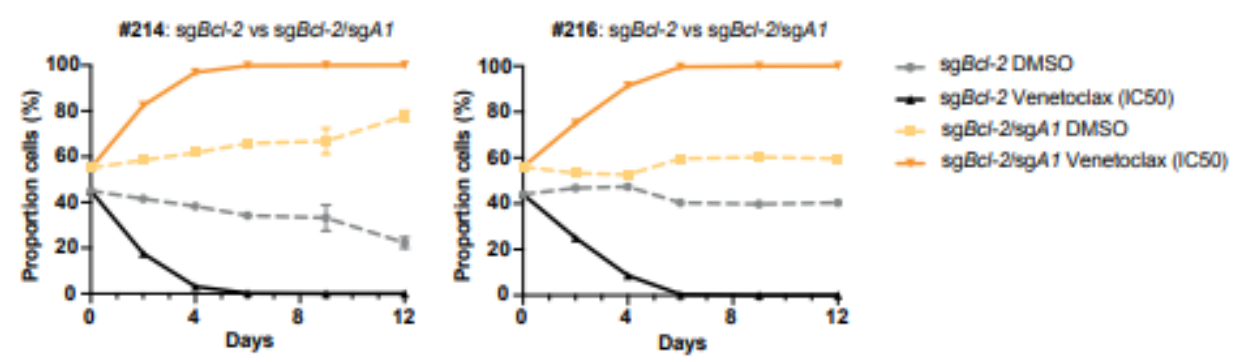

\section{Figure 6}

Whole genome CRISPR activation screens in high MYC/BCL-2 expressing lymphoma-derived cell lines reveal upregulation of $\mathrm{A} 1$ as a dominant resistance factor for venetoclax treatment. a Schematic of whole genome CRISPR activation screens performed in two independently derived murine lymphoma cell lines (\#214, \#216). Six replicates of sgRNA library-infected cells were cultured for 2 weeks in the presence of vehicle (DMSO; control) or the indicated doses of the BCL-2 inhibitor venetoclax before being analysed by next generation sequencing to identify enriched sgRNAs. $b$ Heatmaps showing the proportions of sequencing read results which mapped to sgRNAs targeting the promoter regions of the pro-survival gene A1. The six replicate samples for each treatment condition are shown in columns A-G. c Western blots 
confirming upregulation of A1 protein in DHL cell lines transduced with individual sgRNAs targeting the A1 promoter (sgA1). Probing for HSP70 was used as a loading control. d Competition assays of \#214 or \#216 DHL cells vs the same cell lines carrying an additional sgRNA targeting A1 (sgA1) as shown in c. Cells were mixed 1:1 and treated with DMSO (negative control) or IC50 doses of venetoclax. The contributions of cells of each genotype to the population were monitored over time by flow cytometry.

\section{Supplementary Files}

This is a list of supplementary files associated with this preprint. Click to download.

- SupplementaryData.xlsx

- SupplementaryinformationDeng.pdf 